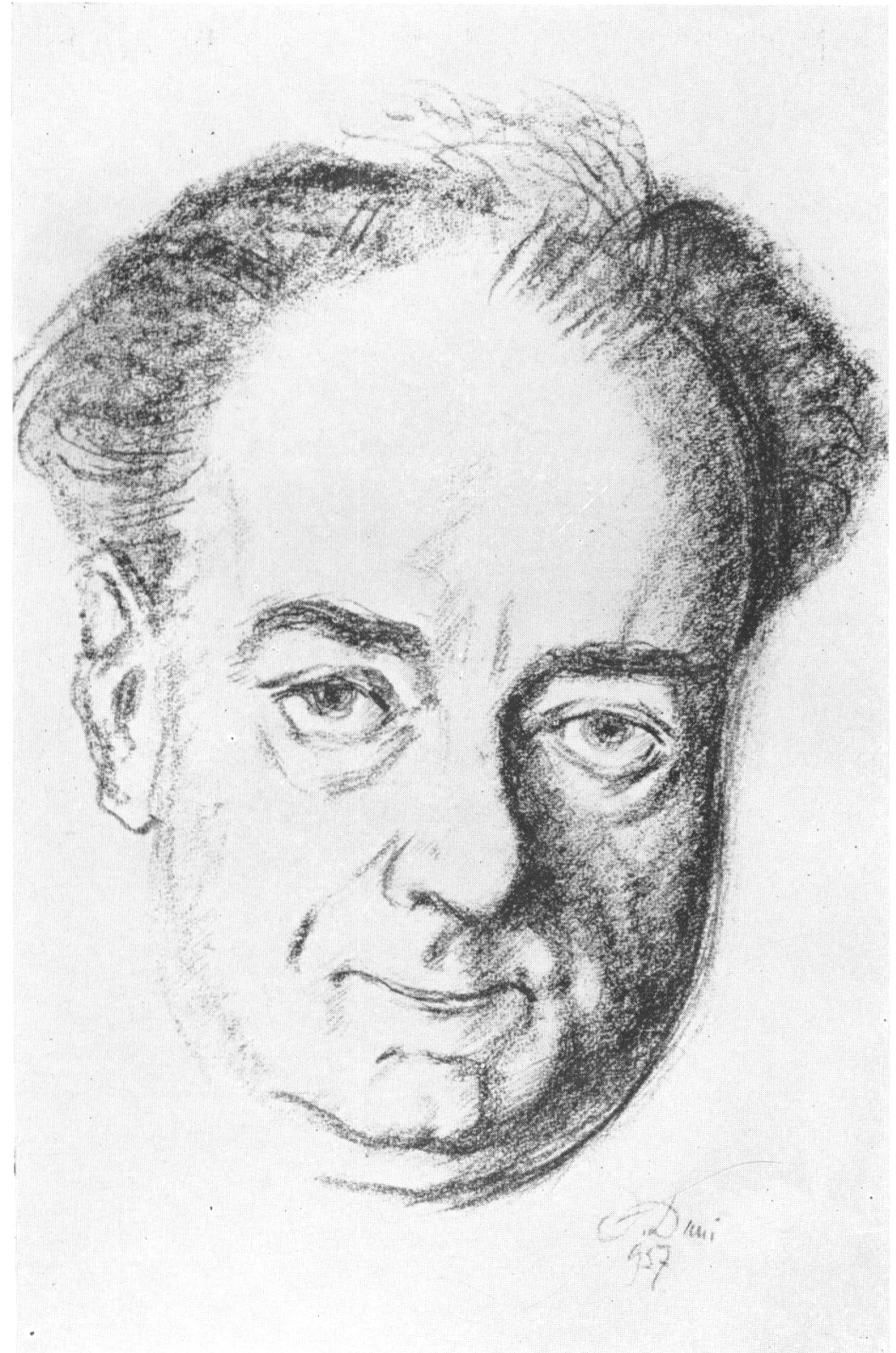

Mrango siport. 


\section{Hommage à Armando Sapori *}

I

L EST, DE PAR IE MONDE, de grands historiens qu'on admire. Les aimer, nul n'y pense.

Il est de bons historiens. On peut les aimer ; mais les admirer, on ne saurait.

Il est enfin quelques grands historiens qu'on admire et qu'on aime. Tel, hier, le cher, le vaillant et toujours regretté Hènri Pircnne. Tel, aujourd'hui, le cher, le généreux Armando Sapori. On l'aime autant qu'on l'admire. Notre éminent collègue Gino Luzzatto, avec son autorité, dira les raisons de notre admiration. Je voudrais pouvoir dire celles de notre affection.

La réponse sans doute pourrait tenir en un mot. On aime l'auteur des Studi medievali parce qu'il est aimable. Qui, pour la première fois, se rend à son appel, pourrait s'attendre, après tout, à rencontrer quelque vénérable savant dont le corps et l'esprit retiendraient un peu de cette poussière subtile que dégagent les vieux dossiers d'archives. Un docte professeur, bien professoral. Serré dans un vêtement noir, un peu fatigué aux genoux et aux coudes. Sachant tout, et ne le laissant ignorer à personne. Bref, un robot d'histoire avant la lettre. Et voilà. La porte s'ouvre. Un homme se dresse. Un homme fort, aux mains tendues, au regard vif et bon et qui, lorsqu'il accueille un visiteur dont il se promet quelque plaisir, lui sourit d'un large et franc sourire. Un homme, cette chose splendide et rare. Pourquoi continuer? D'un mot, on aime Armando Sapori parce qu'on le sent, dès l'abord, prêt à vous aimer. Et que cet élan spontané vous donne le goût de lui plaire et la joie de lui parler.

Est-ce tout cependant, et peut-on s'arrêter là ? Tout bon fils de Clio sait qu'il convient d'analyser avant de faire synthèse. Chaque réussite humaine pose un problème, et des plus compliqués. C'est ainsi précisément que, s'enrichissant, la recherche de l'historien part à la rencontre des recherches voisines : la géographique, la sociologique, la psychologique, - voire la psychanalytique. Si bien que, s'agissant de notre ami, nous nous sentirions un peu gênés sans doute pour instaurer une telle

* Les Annales empruntent aux Studi in onore di $A$. Sapori, ce beau portrait d'historien tracé par Lucien Febvre au printemps de 1956 : façon d'honorer, à notre tour, le grand historien italien. 
minutieuse enquête s'il ne nous avait fait don d'un étonnant " Guide par Sapori à travers Sapori n. Il l'a intitulé Mondo Finito ${ }^{1}$. Un chef-d'œuvre. Intraduisible, j'en ai peur. Il faudrait pour le transposer en français un maître écrivain aussi habile à mobiliser toutes les ressources de son parler d'Ile-de-France que notre auteur à manier sa belle langue toscane. Il faudrait, par surcroît, un psychologue qui ait reçu en don assez de sensibilité, de pudeur et d'esprit pour ne trahir en rien ce livre si plein de talent, si subtilement délicat, si débordant d'humanité, - et qui, strictement individuel d'intention, n'en porte pas moins témoignage pour tout un peuple : mieux, pour toute une civilisation.

Armando Sapori n'est pas né sur les degrés d'une chaire professorale. Aucune fée n'a déposé dans son berceau un titre doctoral rédigé (en latin) par la divine Providence. Point d'ascendants historiens dans la famille, semble-t-il. Un milieu de petite bourgeoisie ? J'hésite à le dire. Bourgeoisie est un mot si usé qu'il perd son sens de plus en plus, en dépit ou à cause de tant de savants ouvrages consacrés à en obscurcir la notion première. Une famille bourgeoise, même modeste, de cette vaillante et adorable Sienne est sans doute, de par ses conceptions de vie et ses traditions, bien autre chose qu'une famille Yvelot, somnolente dans son gras bien-être normand. Par ailleurs, chez nous, Français, qu'attendrit toujours le qualificatif de "petit" (nos petits soldats, nos petits artisans, voire nos petits bistrots), l'appellation (non contrôlée) de "petit bourgeois " est, par une exception singulière, le contraire d'un compliment dans la bouche de certains. Il est vrai que ceux qui la brandissent avec des gestes d'exorcisme ont quelques raisons, parfois, pour ne point s'accorder avec nos traditions...

Disons simplement qu'on n'était pas riche chez les parents d'Armando Sapori. Du moins, pas riche de ces biens de fortune dont il parle si excellement dans la courte Préface à ses lecteurs de ce qui n'est pas une autobiographie (l'horrible mot, qui sent à la fois la mécanique et la dissection), mais une médiation sentimentale, l'inventaire d'un patrimoine, un document aussi d'histoire naturelle et sociale. Les biens de fortune ? " $\boldsymbol{S i}$ rifanno con pazienza $e$ con sacrificio... $e$ i miei... sapevo che avrebbero ricominciato da capo, con la tenacia che è propria della nostra gente ". Ténacité : le beau mot, par contre! Nous aussi, Franc-Comtois, défricheurs acharnés d'une terre montagnarde sans grand sourire, où les épicéas et les noirs sapins remplacent les hauts cyprès chers à Gozzoli, nous aimons l'appliquer à nos ancêtres et à nous. Ce n'est pas la première fois que je

1. Armando Sapori, Mondo finito, Florence, 1946. 
buterai sur une de ces ressemblances profondes. Expliqueraient-elles pour ma part, au tréfonds de moi-même, l'inclination qui, dès la première rencontre, me fit comprendre et goûter Armando Sapori ? Quoi qu'il en soit, dans la maison paternelle du futur médiéviste, aucun Pactole ne coulait. Et quand le chef de famille eût disparu, trop tôt, un grand poids de responsabilité retomba sur le fils. Heureusement, il était de bonne trempe. Mais enfin, il fallut bien qu'il se préoccupât de son avenir immédiat et que, pour vivre, il répudiât des projets à trop longue échéance.

\section{$\star$}

Pauvreté, dure maîtresse des adolescences volontaires. Pour un Français de mon âge, le thème n'est plus à développer, après Charles-Louis Philippe, ou après Charles Péguy. S'agissant d'Armando Sapori, toute une part de ce qui fait son attrait réside sans doute dans scs débuts difficiles. Il a le charme des hommes qui se sont forgés eux-mêmes, par cet héroïque " travail de soi sur soi " dont parle notre Michelet; Iui non plus n'a pas été au seuil de sa vie (et pour cause !) installé douillettement dans un compartiment de première classe, bien rembourré, en abandonnant les soucis de l'itinéraire au chef de train; à l'arrivée, l'hôtel (Excelsior), de première classe également. A tant d'amollissantes séductions, on peut sans doute s'arracher. En France, de mon temps, les « résistants " de cette sorte n'ont point manqué : Barrès, Gide, pour ne point parler de Proust. Ils n'ont rien à voir avec un Sapori. Chez lui, un refoulement, tout provisoire, de trop vastes desseins. Un obstiné souci de se libérer de la hantise du lendemain. La certitude au fond de lui qu'il sortirait de la gêne et triompherait des obstacles. Bref, ce qui fait un homme. Un homme vraiment homme : nous y revenons toujours.

Pauvreté, pauvreté ? Il ne faudrait rien exagérer pourtant. Quant aux biens de fortune, soit; mais les autres? En dépit de sa délicatesse (qui le fait nous parler longuement de ses grands-parents et de ses oncles, mais non de ses propres parents, trop intimement mêlés à sa substance pour qu'un sentiment raffiné de pudeur ne lui interdise pas ce qui serait confidences et peut-être indiscrétion), en dépit de ces subtiles et rares nuances de sentiment, l'auteur de Mondo Finito nous en dit assez pour que nous puissions pénétrer jusqu'aux sources profondes de sa nature d'homme. Généreux, confiant, aimant construire l'avenir, et pourtant lesté d'un poids d'ordre, de sagesse, de maîtrise de soi capable d'arrêter les excès. Notre P. J. Proudhon, un jour, a défini ce type d'homme ainsi fait : "Anarchistes [le superlatif des " gauches " d'alors], mais anarchistes de gouvernement. "Proudhon l'a dit de ses compatriotes, mes compatriotes franc-comtois, eux aussi fortement lestés de catholicisme traditionnel, imbus d'un sens de l'ordre profondément ancré, mais s'évadant 


\section{ANNALES}

volontiers des cadres hiérarchiques pour imaginer (avec Charles Fourier, avec Victor Considérant, avec Proudhon lui-même) des formes idéales de sociétés dont la liberté ferait la force et la fécondité. Déisme humanitaire des Maçons du XvIII ${ }^{\mathrm{e}}$ siècle (leur représentant à Sienne fut précisément l'aïeul maternel d'Armando Sapori); élans de cœur assaisonnés d'une pointe de Mazzini ; en face, chez la grand-mère, austère rigidité d'un catholicisme durci dans ses croyances et dans ses pratiques : ce mélange de courants en apparence contraires, quand notre historien le retrouve, toujours agissant, au fond de son être, faut-il parler encore de pauvreté, ou bien, - songeant à ceux qui déposèrent en lui les éléments d'une telle chimie intérieure, — d'un précieux héritage de valeurs culturelles ? Celles-là même, finalement, qui firent la grandeur de notre civilisation d'Occident.

Autre chose encore. Ne l'appelons pas, cette chose, "le milieu ». Il ne s'agit pas de brosser, à la vieille mode d'Hippolyte Taine, un décor de théâtre sur quoi se profileraient les personnages du drame humain. Ici encore, il s'agit de participation à des forces profondes et nourricières : celles même de l'histoire, senties par un historien à travers sa nature d'homme. Dans sa patrie nationale, l'Italie, Armando Sapori possède sa patrie à lui, celle qui sans doute alimenta ses rêveries d'adolescent, celle qui l'a fait l'historien qu'il est : je veux dire la Toscane, une et bicéphale. Ici Florence, dont on n'a le droit de parler que si l'on est l'Alighieri, ou Michel Ange. Là Sienne, l'exquise Sienne, avec, comme dit joliment son enfant fidèle, "la sorridente quiete pensosa che la definisce negli affreschi del Pinturicchio n et sur quoi règne, tutélaire et vigilant, le Pinnacolo de la Torre del Mangia. Florence, Sienne, la Toscane : l'une des deux ou trois plus belles patries culturelles que puisse désirer un Européen conscient des trésors dont il est le détenteur temporaire. Florence, Sienne, la Toscane : l'une des contrées de l'univers les plus fourmillantes d'histoire toujours vivifiante, l'un des berceaux les plus riches en génies de notre monde civilisé.

Combien cette terre de ses ancêtres a pu, a dû exalter la sensibilité d'un homme qui, comme tous les vrais historiens, n'étudie pas l'histoire mais la vit, - je l'ai compris un jour, au début de mes relations avec Armando Sapori. Il venait d'arriver à Paris, qu'il ne connaissait pas. "Voulez-vous que demain matin j'aille vous prendre pour faire avec vous un tour d'horizon ? " «Oh ! merci, me répondit-il avec une sorte de confusion pudique ; merci, mais demain je me suis juré d'aller, avant tout, rue des Lombards. Je veux [voir et son accent disait : je veux voir seul] ces maisons, ces rues où tant de mes ancêtres sont venus travailler pour leur pays, et pour le vôtre aussi..." 
Je ne lui dis pas qu'en fait de "Lombards ", il risquait fort de ne croiser, au cours de sa promenade, que d'étranges silhouettes d'Orientaux à longues barbes bouclées, étalées sur des lévites noires. Notre pèlerin les vit sans aucun doute dans le cadre médiéval qu'ils adaptent quotidiennement à leurs besoins. Mais j'imagine qu'il vit surtout, avec les yeux de son imagination et de son cœur, "ses ancêtres " comme il disait : ceux à l'activité novatrice desquels il avait consacré sa vie d'historien.

Tiendrions-nous cette fois, isolés par l'analyse, tous les éléments d'un complexe humain dont on ne découvre les richesses que lentement ? Non pas. Dans une lettre déjà ancienñe (décembre 1952), notre ami rappelait une boutade de moi, écrite je ne sais plus quand, et qui l'avait frappé : "Tout historien véritable a deux patries, le passé et le présent. " Sur quoi, il développait : "Homme d'étude, je ne m'intéresse pas à la politique comme à une activité sans lien avec la science; je pars de mes réflexions sur le passé pour m'orienter à travers la vie d'aujourd'hui : "... parto dalla riflessione sul passato per orientarmi nella vita di oggi ".

En fait, notre ami s'est mêlé activement à la politique de son pays. Il ne s'en était jamais désintéressé. Tout jeune, à Sienne, il avait fait ses premières armes de journaliste dans une feuille locale, la Vedetta Senese. Après l'effondrement du régime fasciste - contre lequel il mena une opposition d'esprit et une polémique d'humour qui auraient pu le conduire loin - il accepta et occupa, de 1948 à 1953, un siège de sénateur indépendant de gauche qui lui permit de participer activement au travail des Commissions, de prononcer quelques discours remarqués et, surtout, de témoigner par sa présence et par ses votes de son ardent désir de ne pas perdre contact avec les masses populaires et prolétariennes, de s'unir à ceux qui défendaient leur cause. On lui a reproché cette activité. Certains de ses amis redoutaient, j'imagine, qu'il perdît trop de temps dans cette carrière nouvelle. Je n'ai certes pas à prendre parti dans un débat qui d'ailleurs ne regarde que lui. Mais il me sera permis, en homme qui n'a jamais voulu faire de politique par insurmontable aversion pour les embrigadements, de dire ceci : lorsqu'on est Armando Sapori, on ne perd jamais son temps. Ce n'est pas perdre son temps que de sortir de son trou d'érudit - Hans im Schnokeloch, disent les Alsaciens dans leur dialecte - pour participer au jeu d'une grande politique nationale, s'initier au mécanisme des partis, à l'occasion contempler "leurs Figures " comme disait Barrès dans son livre sur le scandale de Panama, un des chefs-d'œuvre de la littérature partisane en France. Ni l'historien (le véritable historien), ni ceux qui se considèrent comme ses amis ne doivent craindre qu'il ne salisse, en se mêlant aux luttes politiques, sa blanche hermine 
d'érudit. On ne construit pas, on ne construit plus de tours d'ivoire. Et l'histoire, disons $\mathrm{du} \times \mathrm{xv}^{\mathrm{e}}, \mathrm{du} \mathrm{xv}^{\mathrm{e}}, \mathrm{du} \mathrm{xvI}^{\mathrm{e}}$ siècle, ne ressemble pas plus que notre histoire contemporaine à un jardin de Paradis peint par Fra Angelico et peuplé de rondes d'anges et de bienheureux couronnés de lys et de roses. L'histoire de tous les siècles et de tous les peuples est pleine de vols et de viols, de stupres et de meurtres, d'assassinats et de guerres, - bref, de charniers écœurants. Il faut à l'historien, pour s'y promener, un cœur sans défaillance, un odorat sans susceptibilité et des bottes sans trous. Ne faisons done point la petite bouche. Participer au jeu politique d'un grand pays, c'est élargir son expérience humaine. C'est se rendre plus apte à comprendre un immense secteur du passé. C'est vivre d'une vie plus large et plus féconde. Et vivre, pour l'historien, c'est le devoir primordial. Il n'est qu'un pauvre homme d'érudit, un triste " grabeleur de fiches ", s'il doit craindre de se mouiller les plumes en volant à travers la bourrasque. Or, Armando Sapori n'est pas un pauvre homme. C'est un historien, un vrai. Parce qu'il a publié tant de documents ardus, tant de chefs-d'œuvre d'erudition parfaitement achevés ? Certes. Mais aussi parce qu'il a écrit Mondo Finito, qu'il était seul à pouvoir écrire, forme et fond. Parce qu'à Paris, à la VIe Section de l'Ecole des Hautes Etudes, il nous a donné ce portrait si émouvant du marchand italien du moyen âge, qu'il n'avait pas besoin de signer pour qu'on en reconnût l'auteur. Et parce que, fils de ses ouvres et ne l'oubliant pas, il a toujours pensé, sans reniement, à ceux qui, moins que lui encore, n'ont pas joui de cette sécurité matérielle qui engendre la sécurité morale.

Et puis, et puis... Nous allons disant volontiers (nous du moins, Français) : " Nos parlementaires ne sont pas de taille à remplir comme il faudrait leurs fonctions de plus en plus énormes »... Alors, soyons logiques. Et n'incriminons pas les hommes d'étude qui font le nécessaire pour remédier, personnellement, à cette carence. Ambition, ambition : mot vite dit. Je réponds, avec mon ami l'historien : "Non sarei uomo se non avessi ambizioni. E non sarei galantuomo se non avessi ambizioni oneste " (Avril 1952).

Aussi bien, la question politique ne se pose plus : pour des raisons plus qu'honorables, Armando Sapori n'est point resté sénateur de la République. Mais, dans l'énergie qu'il déploie aujourd'hui en faveur des jeunes gens, dans l'effort qu'il poursuit à la tête de la plus grande université commerciale d'Italie pour renouveler l'enseignement que réclament les jeunes, pour les loger et les nourrir, pour les guider surtout et les soutenir dans leurs études, - qui donc dira que l'expérience acquise de 1948 à 1953 par le sénateur Armando Sapori n'ait pas encore élargi ses vues et son esprit, fait tomber chez lui quelques préjugés d'homme de livres et de bibliothèques, confirmé sa confiance dans son sens humain ? La vie ne se condense pas en pilules : un gramme de moyen âge, deux de $\mathrm{xvI}^{\mathrm{e}}$ siècle 
et trois de XviIIe. Elle comprend tout, anime tout; elle circule dans nos esprits comme le feu dont parle notre Rabelais, - le feu qui court à travers les brandes, "infatigable et strident".

Et maintenant, résumons. Un homme qui a dû se faire lui-même. Autodidacte, écrit-il dans la récente Préface de ses Studi di storia economica medievale. Un homme en qui se heurtaient deux styles de vie radicalement opposés, entre lesquels il a dû sans cesse négocier de mouvants concordats : ici, un catholicisnıe fermé ; là, un humanitarisme ouvert. Un homme qui a eu la chance de naître à Sienne, de vivre à Florence, de nourrir sur cette terre d'histoire unique "la passione, comme il me l'écrivait un jour, e insieme il rispetto per la storia ". Un archiviste qui n'a pas considéré que toute sa vie dût consister à se faire l'archiviste de lui-même, à tuer en lui les sentiments, à refouler si bien leur expression qu'ils périssent finalement, desséchés et racornis. Regardez attentivement notre ami, tel que vous le voyez vivre et agir : il n'est rien en lui, il n'est rien de lui que vous ne sachiez expliquer par ces quelques traits.

Le voilà, chez lui, à Florence... Où voulez-vous qu'il habite, sinon Piazza Santa Maria Novella, un des lieux les plus fameux de l'Athènes toscane ? De ses fenêtres, il voit l'église dont le cloître abrite les étonnantes fresques qui résument tout l'ordre spirituel du moyen âge, et le chœur, de par la grâce de Ghirlandaio, toute la noblesse de vie du Quattrocento. Mais sur le parvis du temple, à la Libération, en rentrant chez lui, l'historien a vu également huit cadavres tout chauds encore, dont celui d'une femme : tristes victimes des atroces tragédies que nous avons dû vivre, nous tous Européens qui n'inclinons pas la liberté devant la force brute. J'imagine qu'en traversant la Piazza notre ami y pense souvent et puise dans ce souvenir lugubre plus de raisons encore d'aimer la Paix, en homme fort, sans bêlement enfantin.

Le voilà donc, à Florence, dans ce cabinet de travail fait à sa mesure et qui de cent façons témoigne de ses remarquables aptitudes d'artisan. Artisan en reliure, artisan en imprimerie, artisan en photographie, aussi capable d'habiller de cuir, dans le style du temps, un manuscrit médiéval que de tirer sur les presses une feuille d'imprimerie par lui composée à la main, à la façon des vieux maîtres. Artisan qui aime les artisans, qui sait les écouter de façon unique, dont l'attention et l'affection pressentie encourage ces simples gens à se livrer, ces chères petites gens au grand cœur, au langage souvent exquis dont tomba amoureux notre Anatole France du temps qu'il écrivait Le Lys rouge. A Florence : mais ils en ont pareillement à Sienne. Et combien de fois le petit Armando n'a-t-il pas joué avec leurs fils dans les rues sombres et fraîches de la ville de 


\section{ANNALES}

Duccio, sous l'égide du "Bon Gouvermement " ? Après tout, n'est-il pas resté l'un d'entre eux, même quand, sous la lampe, attentif et scrupuleux, il étudie scientifiquement son admirable collection de timbres ou compose une de ces étonnantes merveilles que sont les tables de ses grandes publications...

Oui, le voilà chez lui. Solitaire ? Non pas. Le méchant vit toujours seul, professaient nos humanitaires du XVIII ${ }^{\mathbf{e}}$ siècle, ces maîtres en sociabilité. Armando Sapori n'a rien du " méchant ". Et comment - si discret qu'on souhaite de demeurer - comment taire cet aspect de sa vie ? Mais en parler... Assurons-nous de tenir le langage qui convient en l'empruntant à une plume irrécusable, celle du Président Einaudi, " un de ces grands bourgeois honnêtes de la vieille souche ", comme me l'écrivait l'éditeur des Fonti, en avril 1952, dans un de ces mouvements de cœur qui l'honorent. Dans la Préface dont il a voulu honorer l'un des récents travaux de l'historien, il rappelle, d'une façon qui a beaucoup touché notre ami, la part que prirent successivement, à l'établissement du Livre des Peruzzi (1934), puis -des documents qui font revivre l'activité des Alberti Del Guidice (1952), et sa femme - que je n'ai pas connue - et sa fille dont personne n'ignore l'énergie, l'intelligence, le total dévouement à la personne et à l'œuvre de son père. L'épouse et la fille, écrivait l'éminent préfacier, " moglie e figlia sono una cosa sola per il curatore dell'opera ". Aussi bien, un Sapori sevré de pareilles affections, un Sapori contraint de vivre dans un elimat d'indifférence intellectuelle et morale, cesserait d'être lui-même. Ce ne sont pas ses amis seuls, ce sont tous les amis de l'histoire qui doivent entourer de respect et de gratitude celles qui auraient pu se borner à porter un grand nom sans le mériter quotidiennement par le plus noble effort.

Ai-je tout dit cette fois ? Peut-être s'étonnera-t-on que je n'aie pas marqué avec plus de force l'originalité d'une carrière qui dut beaucoup moins aux leçons $e x$ cathedra des maîtres d'une Université qu'à l'apprentissage que fit, aux Archives de Florence, à même les dossiers et les liasses, mais au contact de compagnons d'élite (Panella, Anzilotti, Barbadoro), le futur éditeur de tant de documents précieux. Initiation qui, pour un historien français évoque le grand souvenir de Jules Michelet, - mais, pour un historien italien, j'imagine, le caractère d'abord tout municipal d'une formation scientifique qui est allée sans cesse en s'élargissant. Mais ceci, son illustre compagnon d'armes, Gino Luzzatto, le dira sans doute, et Sapori lui-même l'a dit excellemment, tant dans Mondo Finito que, récemment, dans la réédition de ses Studi. Cette marche a des horizons plus vastes qu'une recherche limitée et qu'un commentaire également 
limité ; ce désir de brosser de vastes panoramas, soutenus par la connaissance la plus minutieuse des faits, d'autres que lui l'ont éprouvé. Et de nouveau je citerai ici le nom d'Henri Pirenne. Processus normal... pour de vrais historiens.

Faut-il un mot de conclusion ? Notre ami lui-même le dictera. Dans une lettre qu'il m'écrivait le 4 avril 1953, il formulait ainsi son idéal : " realmente indipendente e galantuomo."

C'est le mien. Le nôtre. Celui des hommes qui, incapables de mentir à eux-mêmes comme aux autres, voudraient dans l'affreuse tourmente qui les bat, sans se refuser cependant à aucun effort de compréhension et de sympathie, sauvegarder les valeurs essentielles et les traditions plus que millénaires de l'Occident. A commencer par ce sens de la mesure et cette horreur du colossal - sia americano, sia orientale - que l'historien florentin dénonçait un jour, non sans véhémence. Que sa vie s'achève dans la paix de ces belles espérances. Qu'il puisse préserver jusqu'au bout son indépendance tutélaire. Qu'il garde intacte cette chalcur de cœur qui anime tout ce qu'il dit, tout ce qu'il fait, tout ce qu'il touche : c'est le vœu fervent que, groupés en pensée autour de lui, nous tous - ses aînés comme ses contemporains, ses émules et ses disciples, ses compatriotes comme ses amis d'outre-monts - nous formulons avec, comme il aime à le dire, la plus humaine et la plus fervente des fraternités.

Lucien Febvre. 